\title{
Search for the $S=+1$ pentaquarks in quenched lattice QCD
}

\section{Toru T. Takahashi*}

Yukawa Institute for Theoretical Physics, Kyoto university, Kyoto 606-8502, Japan

E-mail: ttoru@yukawa.kyoto-u.ac.jp

\section{Takashi Umeda}

Yukawa Institute for Theoretical Physics, Kyoto university, Kyoto 606-8502, Japan

E-mail: tumeda@yukawa.kyoto-u.ac.jp

\section{Tetsuya Onogi}

Yukawa Institute for Theoretical Physics, Kyoto university, Kyoto 606-8502, Japan

E-mail: onogi@yukawa.kyoto-u.ac.jp

\section{Teiji Kunihiro}

Yukawa Institute for Theoretical Physics, Kyoto university, Kyoto 606-8502, Japan

E-mail: kunihiro@yukawa.kyoto-u.ac.jp

We study spin $\frac{1}{2}$ hadronic states in quenched lattice QCD to search for a possible $S=+1$ pentaquark resonance. Our work is the first systematic lattice QCD study which properly carries out the following analyses: (1) the careful extraction of the first two low energy states with very high statistics and the variational method and (2) the study of volume dependences of eigenenergies and spectral weights to distinguish resonance states from scattering states.

Simulations are carried out on $8^{3} \times 24,10^{3} \times 24,12^{3} \times 24$ and $16^{3} \times 24$ lattices at $\beta=5.7$ with the standard plaquette gauge action and the Wilson quark action. Our result indicates the existence of a resonance state lying slightly above the NK threshold in $\left(I, J^{P}\right)=\left(0, \frac{1}{2}^{-}\right)$channel in quenched QCD.

XXIIIrd International Symposium on Lattice Field Theory

25-30 July 2005

Trinity College, Dublin, Ireland

\footnotetext{
*Speaker.
} 


\section{Introduction}

After the first discovery [1] of $\Theta^{+}(1540)$, identifying the properties of the particle is one of the central problems in hadron physics. While the isospin of $\Theta^{+}$is likely to be zero, the spin and the parity and the origin of its tiny width still remain open questions. In spite of many theoretical and experimental studies on $\Theta^{+}$[2], the nature of this exotic particle, including the very existence of the particle, is still controversial. In such a situation, the lattice QCD calculation is considered as one of the most reliable methods, which is an ab initio method directly based on QCD.

Up to now, several lattice QCD studies have been reported, which mainly look for pentaquarks in various different ways $[3,4]$. However, the conclusion has not been unfortunately settled yet. The difficulties in the spectroscopy calculation with lattice QCD generally arise from systematic errors due to the discretization, the chiral extrapolation, the quenching effect, the finite volume effect and the contaminations from higher excited-states. The difficulty specific to the current problem is that the signal of $\Theta^{+}$is embedded in the discrete spectrum of NK scattering states in finite volume. In order to verify the existence of a resonance state, one needs to isolate the first few low energy states including the lowest NK scattering state, identify a resonance state and study its volume dependence which can distinguish itself from other scattering states. Ideally, one should extract multistates from a high statistics unquenched calculation for several different physical volumes, where both the continuum and the chiral limits are taken. However, there are no lattice QCD study which performs all these steps so far, due to the enormous computational costs needed. We therefore concentrate ourselves on the analyses using rather heavy quarks on coarse quenched lattices but with a good statistics and a proper separation of states.

In this report, we study $(I, J)=\left(0, \frac{1}{2}\right)$ channel in quenched lattice QCD to search for possible resonance states. We prepare thousands of gauge configurations in order to single out the signals of a resonance state with good statistics. We adopt two independent operators with $I=0$ and $J=\frac{1}{2}$ and diagonalize the $2 \times 2$ correlation matrices for all the combinations of lattice sizes and quark masses to extract the 2nd-lowest state slightly above the NK threshold in this channel. After the careful separation of the states, we investigate the volume dependence of the energy as well as the spectral weight [5] of each state so that we can distinguish the resonance signal from the background signals of NK scattering states. Our calculation (For the details, please see our recent paper, Ref. [4].) gives the first systematic study which performs

- the analysis using correlation matrices with very high statistics, which enables us to isolate the first few low energy states including the lowest NK scattering state

- the study of the volume dependences of eigenenergies and spectral weight factors with several different physical volumes, which enables us to distinguish a resonance state from scattering states

in a proper way.

\section{Setup}

We adopt the variational method using correlation matrices constructed from independent operators [4] in order to isolate the signal of a resonance state from the NK scattering states. The sim- 
ulations are carried out on four different sizes of lattices, $8^{3} \times 24,10^{3} \times 24,12^{3} \times 24$ and $16^{3} \times 24$ with 2900, 2900, 1950 and 950 gauge configurations, respectively, using the standard plaquette (Wilson) gauge action at $\beta=5.7$ and the Wilson quark action. The hopping parameters for the quarks are $\left(\kappa_{u, d}, \kappa_{s}\right)=(0.1600,0.1650),(0.1625,0.1650),(0.1650,0.1650),(0.1600,0.1600)$ and $(0.1650,0.1600)$, which correspond to the current quark masses $\left(m_{u, d}, m_{s}\right) \sim(240,100),(170,100)$, $(100,100),(240,240)$ and $(100,240)$, respectively in the unit of MeV. The lattice spacing $a$ determined from the Sommer scale is about $0.17 \mathrm{fm}$, which implies the physical lattice sizes are $1.4^{3} \times 4.0 \mathrm{fm}^{4}, 1.7^{3} \times 4.0 \mathrm{fm}^{4}, 2.0^{3} \times 4.0 \mathrm{fm}^{4}$ and $2.7^{3} \times 4.0 \mathrm{fm}^{4}$. For the correlation matrices, we adopt two wall-type operators $\bar{\Theta}_{\text {wall }}^{1}(t)$ and $\bar{\Theta}_{\text {wall }}^{2}(t)$ [4], whose spinor and color structures are the same as those adopted in the first paper by Csikor et al. Periodic boundary conditions are taken in all directions for the gauge field, whereas we impose periodic boundary conditions on the spatial directions and the Dirichlet boundary condition on the temporal direction for the quark field in order to avoid possible contaminations from those propagating beyond the boundary at $t=0$ in (anti)periodic boundary conditions [4], which is peculiar to pentaquarks and has not been dwelled.

\section{Lattice QCD Result in $\left(I, J^{P}\right)=\left(0, \frac{1}{2}^{-}\right)$channel}

\section{1 volume dependence of eigenenergies}
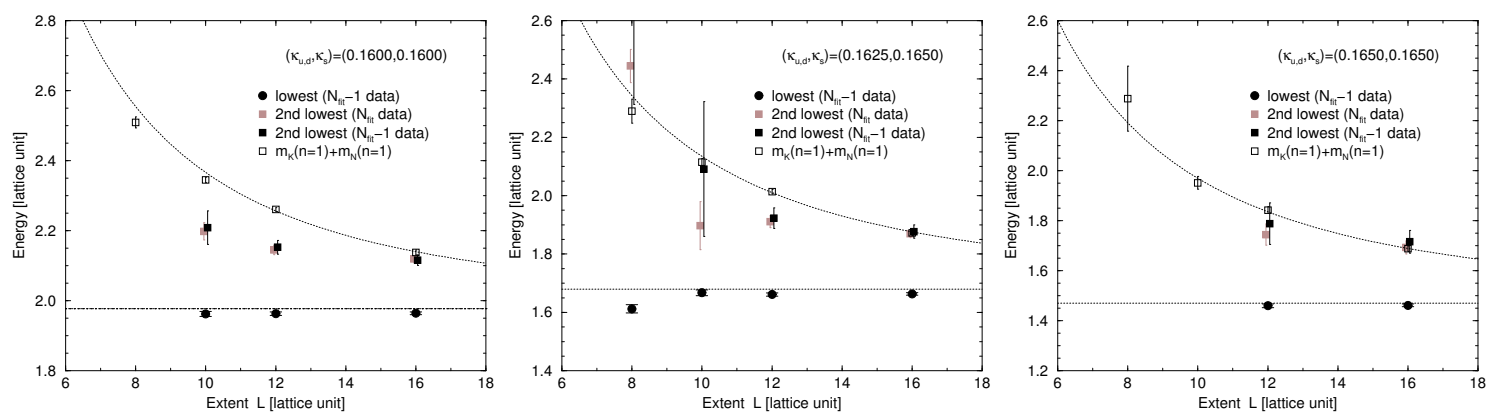

Figure 1: The black (gray) filled-squares denote the lattice QCD data of the 2nd-lowest state in $\left(I, J^{P}\right)=$ $\left(0, \frac{1}{2}^{-}\right)$channel extracted with $N_{\text {fit }}-1\left(N_{\text {fit }}\right)$ data plotted against the lattice extent $L$. The filled circles represent the lattice QCD data $E_{0}^{-}$of the lowest state in $\left(I, J^{P}\right)=\left(0, \frac{1}{2}^{-}\right)$channel. The open symbols are the sum $E_{N}^{|\overrightarrow{\mathbf{n}}|=1}+E_{K}^{|\overrightarrow{\mathbf{n}}|=1}$ of energies of nucleon $E_{N}^{|\overrightarrow{\mathbf{n}}|=1}$ and Kaon $E_{K}^{|\overrightarrow{\mathbf{n}}|=1}$ with the smallest lattice momentum $|\overrightarrow{\mathbf{p}}|=\frac{2 \pi}{L}|\overrightarrow{\mathbf{n}}|=2 \pi / L$. The upper line represents $\sqrt{M_{N}^{2}+|\mathbf{p}|^{2}}+\sqrt{M_{K}^{2}+|\mathbf{p}|^{2}}$ with $|\mathbf{p}|=2 \pi / L$ the smallest relative momentum on the lattice. The lower line represent the simple sum $M_{N}+M_{K}$ of the masses of nucleon $M_{N}$ and Kaon $M_{K}$. We adopt the central values of $M_{N}$ and $M_{K}$ obtained on the largest lattice to draw the two lines.

The filled circles in Fig. 1 show the lowest-state energies $E_{0}^{-}$in $I=0$ and $J^{P}=\frac{1}{2}^{-}$channel on four different volumes. Here the horizontal axis denotes the lattice extent $L$ in the lattice unit and the vertical axis represents the energy of the state. The lower line denotes the simple sum $M_{N}+M_{K}$ of the nucleon mass $M_{N}$ and Kaon mass $M_{K}$ obtained with the largest lattice. We here simply use $M_{N}+M_{K}(L=16)$ as a guideline.

As for the lowest state of this channel, we find that the energy of the state takes almost constant value against the volume variation and coincides with the simple sum $M_{N}+M_{K}$. It is then concluded 
that the lowest state in $I=0$ and $J^{P}=\frac{1}{2}^{-}$channel is the NK scattering state with the relative momentum $|\mathbf{p}|=0$. The good agreement with the sum $M_{N}+M_{K}$ implies the weakness of the interaction between $\mathrm{N}$ and $\mathrm{K}$.

The $\left(I, J^{P}\right)=\left(0, \frac{1}{2}^{-}\right)$state is one of the candidates for $\Theta^{+}(1540)$. Since $\Theta^{+}(1540)$ is located above the NK threshold, it would appear as an excited state in this channel. The volume dependence of the energy of each state can be used to distinguish a possible resonance state from NK scattering states; it is expected that the energies of resonance states have small volume dependence, whereas the energies of NK scattering states are expected to scale according to the lattice size $L$.

A possible candidate for the volume dependence of the energies of NK scattering states is the simple formula as $E_{\mathrm{NK}}^{\overrightarrow{\mathbf{n}}}(L) \equiv \sqrt{M_{N}^{2}+\left|\frac{2 \pi}{L} \overrightarrow{\mathbf{n}}\right|^{2}}+\sqrt{M_{K}^{2}+\left|\frac{2 \pi}{L} \overrightarrow{\mathbf{n}}\right|^{2}}$ with the relative momentum $\frac{2 \pi}{L} \overrightarrow{\mathbf{n}}$ between $\mathrm{N}$ and $\mathrm{K}$ in finite periodic lattices. There may be some corrections to $E_{\mathrm{NK}}^{\overrightarrow{\mathbf{n}}}(L)$ in practice. We therefore estimate three possible corrections [4]; the existence of the NK interaction, the application of the momenta on a finite discretized lattice and the estimation of the implicit finite-size effects. We find that these corrections lead to at most a few $\%$ deviations from $E_{\mathrm{NK}}^{\overrightarrow{\mathbf{n}}}(L)$ and we neglect these corrections for simplicity and use the simple form $E_{\mathrm{NK}}^{\overrightarrow{\mathbf{n}}}(L)$ in the following discussion.

We now compare the lattice data $E_{1}^{-}$with the expected behaviors $E_{\mathrm{NK}}^{|\overrightarrow{\mathbf{n}}|=1}$ for the 2nd-lowest NK scattering states. The filled squares in Fig. 1 denote $E_{1}^{-}$, the 2nd-lowest-state energies in this channel. (The black and gray symbols are the lattice data obtained by the fits with two different fit ranges, which help the readers to see the fit-range dependences.) The upper line shows $E_{\mathrm{NK}}^{|\overrightarrow{\mathbf{n}}|=1}$ estimated with the next-smallest relative momentum between $\mathrm{N}$ and $\mathrm{K}$, and with the masses $M_{K}$ and $M_{N}$ extracted on the $L=16$ lattices. Although the lattice QCD data $E_{1}^{-}$and $E_{\mathrm{NK}}^{|\overrightarrow{\mathbf{n}}|=1}$ almost coincide with each other on the $L=16$ lattices, which one may take as the characteristics of the 2nd-lowest scattering state, the data $E_{1}^{-}$do not follow $E_{\mathrm{NK}}^{|\overrightarrow{\mathbf{n}}|=1}$ in the smaller lattices. Especially when the quarks are heavy, composite particles would be compact objects and show smaller finite volume effects besides those arising from the lattice momenta $\overrightarrow{\mathbf{p}}=\frac{2 \pi}{L} \overrightarrow{\mathbf{n}}$. The statistical errors are also well controlled for the heavy quarks. Thus, the significant deviations in $1.5 \lesssim L \lesssim 3 \mathrm{fm}$ with the combination of the heavy quarks, such as $\left(\kappa_{u, d}, \kappa_{s}\right)=(0.1600,0.1600)$, are reliable and the obtained 2nd-lowest states are difficult to be explained as the NK scattering states. One can understand this behavior with the view that the 2nd-lowest state is a resonance state rather than a scattering state. In fact, while the data with the lighter quarks have rather strong volume dependences which can be considered to arise because of the finite size of a resonance state, the lattice data exhibit almost no volume dependence with the combination of the heavy quarks especially in $1.5 \lesssim L \lesssim 3 \mathrm{fm}$, which can be regarded as the characteristic of resonance states.

\section{2 volume dependence of weightfactors}

For further confirmation, we investigate the volume dependence of the spectral weight [5]. In the case when a correlation function is constructed from a point-source and a zero-momentum point-sink, the weight factor $W_{i}$ takes an almost constant value if $|i\rangle$ is the resonance state where the wave function is localized. If the state $|i\rangle$ is a two-particle state, the situation is more complicated. Nevertheless, when the interaction between the two particles is weak, which is the case in the present situation, the weight factor is proportional to $\frac{1}{V}$. When a source is a wall-type operator $\bar{\Theta}_{\text {wall }}\left(t_{\mathrm{src}}\right)$ as taken in this work, a definite volume dependence of $W_{i}$ is not known. Therefore, we 
re-examine the lowest state and the 2nd-lowest state in $\left(I, J^{P}\right)=\left(0, \frac{1}{2}^{-}\right)$channel with the hopping parameter $\left(\kappa_{u, d}, \kappa_{s}\right)=(0.1600,0.1600)$ and the locally-smeared source $\bar{\Theta}^{2}$ smear $\left(t_{\text {src }}\right)$ [4], which is introduced to partially enhance the ground-state overlap. We additionally employ $14^{3} \times 24$ lattice for this analysis. We extract the weight factors $W_{0}$ and $W_{1}$ using the two-exponential fit as
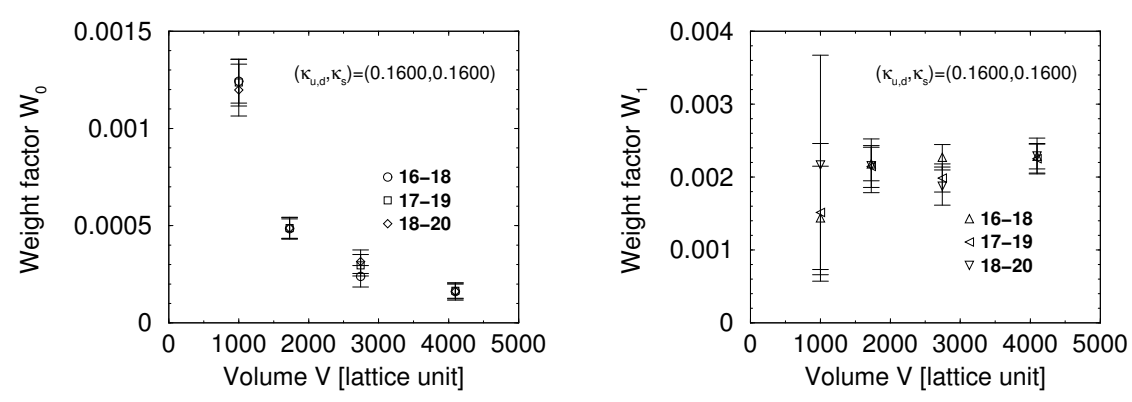

Figure 2: The left figure shows $W_{0}$ for the lowest state in $\left(I, J^{P}\right)=\left(0, \frac{1}{2}^{-}\right)$channel and the right figure shows $W_{1}$ for the 2nd-lowest state. In the case when the weight factor $W_{i}$ for the $i$-th state $|i\rangle$ in a point-point correlator shows no volume dependence, $|i\rangle$ is likely to be a resonance state. On the contrary, when the $i$-th state $|i\rangle$ is a two-particle state, $W_{i}$ scales according to $1 / V$.

$\sum_{\overrightarrow{\mathbf{x}}}\left\langle\Theta^{2}\left(\overrightarrow{\mathbf{x}}, T+t_{\text {src }}\right) \overline{\Theta^{2}}\right.$ smear $\left.\left(\overrightarrow{\mathbf{0}}, t_{\text {src }}\right)\right\rangle=W_{0} e^{-E_{0}^{2} T}+W_{1} e^{-E_{1}^{-} T}$, with the exponents $E_{0}^{-}$and $E_{1}^{-}$fixed. We try the various fit ranges as $\left(T_{\min }, T_{\max }\right)=(16,18),(17,19),(18,20)$ to see the fit-range dependences. Fig. 2 includes all the results and one can find that the global behaviors are almost the same among these three.

The figures in Fig. 2 show the weight factors $W_{0}$ and $W_{1}$ of the lowest and next-lowest state in $\left(I, J^{P}\right)=\left(0, \frac{1}{2}^{-}\right)$channel, respectively. We find that the volume dependence of $W_{0}$ on $V$ is consistent with $\frac{1}{V}$, which is expected in the case of two-particle states. On the other hand, $W_{1}$ shows almost no volume dependence against $V$, which is the characteristic of the state in which the relative wave function is localized. This result can be considered as another evidence of a resonance state lying slightly above the NK threshold.

\section{Lattice QCD Result in $\left(I, J^{P}\right)=\left(0, \frac{1}{2}^{+}\right)$channel}

In the same way as $\left(I, J^{P}\right)=\left(0, \frac{1}{2}^{-}\right)$channel, we have attempted to diagonalize the correlation matrix in $\left(I, J^{P}\right)=\left(0, \frac{1}{2}^{+}\right)$channel using the wall-sources $\bar{\Theta}_{\text {wall }}(t)$ and the zero-momentum point-sinks $\sum_{\overrightarrow{\mathbf{x}}} \Theta(\overrightarrow{\mathbf{x}}, t)$. In this channel, the diagonalization is rather unstable and we find only one state except for tiny contributions of possible other states [4]. The data have almost no volume dependence and they coincide with the solid line which represents the simple sum $M_{N^{*}}+M_{K}$ of $M_{N^{*}}$ and $M_{K}$, with $M_{N^{*}}$ the mass of the ground state of the negative-parity nucleon. The state we observe is therefore concluded to be the $N^{*}-K$ scattering state with the relative momentum $|\mathbf{p}|=0$.

\section{Summary}

We have studied the $(S, I, J)=\left(+1,0, \frac{1}{2}\right)$ states on $8^{3} \times 24,10^{3} \times 24,12^{3} \times 24$ and $16^{3} \times 24$ lattices at $\beta=5.7$ using quenched lattice QCD. We have performed a systematic study by (1) the 
variational analysis using correlation matrices with very high statistics and (2) the study of the volume dependences of eigenenergies and spectral weight factors with several different physical volumes in a proper way for the first time. From the correlation matrix of the operators, we have successfully obtained the energies of the lowest state and the 2nd-lowest state in the $\left(I, J^{P}\right)=$ $\left(0, \frac{1}{2}^{-}\right)$channel. The volume dependence of the energies and spectral weight factors show that the 2nd-lowest state in this channel is likely to be a resonance state located slightly above the NK threshold and that the lowest state is the NK scattering state with the relative momentum $|\mathbf{p}|=0$. As for the $\left(I, J^{P}\right)=\left(0, \frac{1}{2}^{+}\right)$channel, we have observed only one state in the present analysis, which is likely to be a $N^{*} K$ scattering state of the ground state of the negative-parity nucleon $N^{*}$ and Kaon with the relative momentum $|\mathbf{p}|=0$.

The chiral-extrapolated value of the eigenenergy $E_{1}^{-}$of the 2nd-lowest state in the negative parity channel is $1.500(52)$ in the lattice unit, which is about $120 \%$ of the NK threshold 1.227(6). This state is rather heavier than the mass of $\Theta^{+}(1540)$ in the real world. One possibility is the systematic errors from the discretization, the chiral extrapolation, or quenching. Another possibility is that the observed 2nd-lowest state might be a signal of a resonance state lying higher than $\Theta^{+}$in the quenched QCD. It is difficult to give a clear explanation at this point and more extensive studies on finer lattices with lighter quark masses in unquenched QCD will be required. However, we can at least conclude that our quenched lattice calculations suggest the existence of a resonance-like state slightly above the NK threshold for the parameter region we have investigated.

\section{acknowledgments}

T. T. T. was supported by the Japan Society for the Promotion of Science (JSPS) for Young Scientists. T. O. and T. K. are supported by Grant-in-Aid for Scientific research from the Ministry of Education, Culture, Sports, Science and Technology of Japan (Nos. 13135213,16028210, 16540243) and (Nos. 17540250), respectively. This work is also partially supported by the $21 \mathrm{st}$ Century for Center of Excellence program. The lattice QCD Monte Carlo calculations have been performed on NEC-SX5 at Osaka University and on HITACHI-SR8000 at KEK.

\section{References}

[1] T. Nakano et al. [LEPS Collaboration], Phys. Rev. Lett. 91, 012002 (2003).

[2] For example, K. Hicks, arXiv:hep-ex/0504027 and references therein.

[3] F. Csikor, Z. Fodor, S. D. Katz and T. G. Kovacs, JHEP 0311, 070 (2003); S. Sasaki, Phys. Rev. Lett. 93, 152001 (2004); T. W. Chiu and T. H. Hsieh, Phys. Rev. D 72, 034505 (2005); N. Mathur et al., Phys. Rev. D 70, 074508 (2004); N. Ishii, T. Doi, H. Iida, M. Oka, F. Okiharu and H. Suganuma, Phys. Rev. D 71, 034001 (2005); B. G. Lasscock et al., Phys. Rev. D 72, 014502 (2005); F. Csikor, Z. Fodor, S. D. Katz, T. G. Kovacs and B. C. Toth, arXiv:hep-lat/0503012; C. Alexandrou and A. Tsapalis, arXiv:hep-lat/0503013; K. Holland and K. J. Juge [BGR (Bern-Graz-Regensburg) Collaboration], arXiv:hep-lat/0504007.

[4] T. T. Takahashi, T. Umeda, T. Onogi and T. Kunihiro, arXiv:hep-lat/0410025; Phys. Rev. D 71, 114509 (2005).

[5] N. Mathur et al., Phys. Lett. B 605, 137 (2005) [arXiv:hep-ph/0306199]. 